\title{
Machine and deep learning methods for radiomics
}

\author{
Michele Avanzo ${ }^{\text {a) }}$ \\ Department of Medical Physics, Centro di Riferimento Oncologico di Aviano (CRO) IRCCS, 33081, Aviano, PN, Italy \\ Lise Wei \\ Department of Radiation Oncology, University of Michigan, Ann Arbor, MI 48103, USA \\ Joseph Stancanello \\ Guerbet SA, Villepinte, France \\ Martin Vallières \\ Medical Physics Unit, McGill University, Montreal, QC, Canada \\ Department of Radiation Oncology, University of California, San Francisco, San Francisco, CA 94143, USA \\ Arvind Rao \\ Department of Radiation Oncology, University of Michigan, Ann Arbor, MI 48103, USA \\ Department of Computational Medicine \& Bioinformatics, University of Michigan, Ann Arbor, MI 48103, USA \\ Olivier Morin \\ Department of Radiation Oncology, University of California, San Francisco, San Francisco, CA 94143, USA \\ Sarah A. Mattonen \\ Department of Radiology, Stanford University, Stanford, CA 94305, USA \\ Issam El Naqa \\ Department of Radiation Oncology, University of Michigan, Ann Arbor, MI 48103, USA
}

(Received 25 February 2019; revised 22 May 2019; accepted for publication 13 June 2019; published 15 May 2020)

Radiomics is an emerging area in quantitative image analysis that aims to relate large-scale extracted imaging information to clinical and biological endpoints. The development of quantitative imaging methods along with machine learning has enabled the opportunity to move data science research towards translation for more personalized cancer treatments. Accumulating evidence has indeed demonstrated that noninvasive advanced imaging analytics, that is, radiomics, can reveal key components of tumor phenotype for multiple three-dimensional lesions at multiple time points over and beyond the course of treatment. These developments in the use of CT, PET, US, and MR imaging could augment patient stratification and prognostication buttressing emerging targeted therapeutic approaches. In recent years, deep learning architectures have demonstrated their tremendous potential for image segmentation, reconstruction, recognition, and classification. Many powerful open-source and commercial platforms are currently available to embark in new research areas of radiomics. Quantitative imaging research, however, is complex and key statistical principles should be followed to realize its full potential. The field of radiomics, in particular, requires a renewed focus on optimal study design/reporting practices and standardization of image acquisition, feature calculation, and rigorous statistical analysis for the field to move forward. In this article, the role of machine and deep learning as a major computational vehicle for advanced model building of radiomics-based signatures or classifiers, and diverse clinical applications, working principles, research opportunities, and available computational platforms for radiomics will be reviewed with examples drawn primarily from oncology. We also address issues related to common applications in medical physics, such as standardization, feature extraction, model building, and validation. (C) 2019 American Association of Physicists in Medicine [https://doi.org/10.1002/mp.13678]

Key words: deep learning, machine learning, quantitative image analysis, radiomics

\section{INTRODUCTION}

Radiomics is an emerging area in quantitative image analysis that aims to relate large-scale data mining of images to clinical and biological endpoints. ${ }^{1}$ The fundamental idea is that medical images are much richer in information than what the human eye can discern. Quantitative imaging features, called also "radiomic features" can provide richer information about intensity, shape, size or volume, and texture of tumor phenotypes using different imaging modalities (e.g., MRI, CT, 
PET, ultrasound, etc.). ${ }^{2}$ Tumor biopsy-based assays provide limited tumor characterization as the extracted sample may not always represent the heterogeneity of the whole patient's tumor, while radiomics can comprehensively assess the threedimensional (3D) tumor landscape by means of extracting relevant imaging information. ${ }^{3}$ It implies that, applying wellknown machine learning methods to radiomic features extracted from medical images, it is possible to macroscopically decode the phenotype of many physio-pathological structures and, in theory, solve the inverse problem of inferring the genotype from the phenotype, providing valuable diagnostic, prognostic, or predictive information. ${ }^{4,5}$

The term radiomics originated from other - omics sciences (e.g., genomics and proteomics) and conveys the clear intent to invoke personalized medicine based on medical images. It traces its roots to computer-aided detection/ diagnosis (CAD) of medical images. ${ }^{6,7}$ However, with recent advances and the diversity of medical imaging acquisition technologies and processing, radiomics is establishing itself as an indispensable image analysis and understanding tool with applications that transcend diagnosis into prognosis and prediction approaches for personalizing patients' management and their treatment. One of the main differentiators from CAD consists of the link that radiomics has to establish between the current features of a physio-pathological structure at the time of investigation and its temporal evolution in order to personalize the therapeutical approach. ${ }^{8}$ The recent availability of large databases of digital medical images and annotated information (e.g., evolution over time or response to treatment with a given prescription, clinical, and survival information), the increase in computational power based on advanced hardware (e.g., GPU, cluster or cloud computing) as well as the tremendous mathematical and algorithmic development in areas like machine or deep learning have created favorable conditions to untap the potential of the enormous amount of imaging data wealth that is being generated.

Certainly, the complementarity of other information such as clinical or laboratory data as well as interaction measurements (e.g., radiogenomics, ${ }^{9}$ relating imaging to genomics, or exposomics, that is the complementary information from the interaction of the patient with environmental variables) will play a key role to drive future success of radiomics, such as accuracy and reproducibility, to levels that are acceptable for routine clinical practice.

Radiomics has been applied to many diseases including cancer and neurodegenerative diseases to name a few. Although the examples drawn here are from the cancer field, the principles presented here are generally universal across the medical imaging domain. The number of publications issued in the last years has grown almost exponentially. Although there are many review articles already about radiomics, its definition, technical details, and applications in different areas of medicine, the view of radiomics as an image mining tool lends itself naturally to application of machine/deep learning algorithms as computational instruments for advanced model building of radiomics-based signatures. ${ }^{9,10}$ This will be the main subject of this article, addressing issues related to common applications in medical physics, standardization, feature extraction, model building, and validation.

\section{OVERVIEW OF RESEARCH AND CLINICAL APPLICATIONS OF CANCER RADIOMICS}

In this section the applications of radiomics to tumor detection and characterization and prediction of outcome will be reviewed. All the studies described are retrospective and mono-institutional, except where noted.

\section{A. Radiomics in diagnosis}

\section{A.1. Cancer detection and auto contouring}

The radiomics approach of combining the extraction of radiomic features with machine learning, can be used either to detect/diagnose cancer or to automatically contour the tumor lesion. Methods for radiomics-driven automatic prostate tumor detection typically use a supervised method trained on a set of features calculated from multimodality images. ${ }^{11}$ For detection of prostate cancer, features were computed in a $3 \times 3$ pixels sliding window in multimodal MRI of prostate. The voxels were tagged as cancerous or noncancerous using a support vector machine (SVM) classifier. ${ }^{12}$ In Algohary et al. ${ }^{13}$, the prostate was segmented into areas according to the aggressiveness between malignant and normal regions in the training groups. A voxelwise random forest model (RF) with a conditional random field spatial regulation was used to classify the voxels in multimodal MRI (T1, Contrast - Enhanced (CE) T1, T2, and FLAIR) of the brain of glioblastoma multiforme (GBM) patients into five classes: nontumor region and four tumor subregions including necrosis, edema, nonenhancing area, and enhancing area ${ }^{14}$ area. $^{12}$ Convolutional neural networks have also been applied to segment organs at risk in head and neck cancer radiotherapy ${ }^{15}$ and in lung ${ }^{16}$ and liver cancers ${ }^{17}$ compared to traditional methods.

\section{A.2. Prediction of histopathology and tumor stage}

Radiomics holds the potential to revolutionize the conventional tumor characterization and replace classic approaches based on macroscopic variables and can be used to distinguish between malignant and benign lesions. ${ }^{3}$ Breast cancer lesions, automatically detected using connected component labeling and adaptive fuzzy region growing algorithm, were classified using radiomic features as benign mass or malignant tumor on digital mammography, ${ }^{18}$ dynamic contrast-enhanced (DCE) MRI, and ultrasound. ${ }^{19}$ A radiomic model based on mean apparent diffusion coefficient (ADC), had better accuracy than radiologist assessment for characterization of prostate lesions as clinically significant cancer (Gleason grade group $\geq 2$ ) during prospective MRI interpretation. ${ }^{20} \mathrm{~A}$ deep learning multiparametric MRI transfer learning method 
has also shown the ability to classify prostate cancer high grade/low or grade. ${ }^{21}$ Radiomic models based on CT images have been used to predict the histopathology (adenocarcinoma or squamous cell carcinoma $)^{22,23}$ and PET tumor stage ${ }^{24}$ of lung cancer as well as micropapillary patterns in lung adercarcinomas. $^{25}$

\section{A.3. Microenvironment and intra-tumor partitioning}

A radiomic signature combining features from CE-CT, and 18F-FDG PET was implemented for the presence of high level of hypoxia in head and neck cancer, defined in terms of maximum tumor-to-blood uptake ratio $>1.4$ in the $18 \mathrm{~F}$ FMISO PET. ${ }^{26}$ Classification and clustering methods have been developed for tumor separation into subregions (habitat imaging), which contributes to the revelation of tumor heterogeneity, and potential selection of subregions to boost radiation dose. ${ }^{27} \mathrm{~A}$ radiomics analysis focused on a characterization of GBM diversity, using various diversity indices to quantify habitat diversity of the tumor as well as to relate it to underlying molecular alterations and clinical outcomes. ${ }^{28}$

\section{A.4. Tumor genotype}

Significant associations between the radiomic features and gene-expression patterns were found in lung cancer patients. ${ }^{3}$ A radiogenomic study demonstrated the associations of radiomic phenotypes with breast cancer genomic features as mitochondrial DNA (miRNA) expressions, protein expressions, gene somatic mutations, and transcriptional activities. In particular, tumor size and enhancement texture had associations with transcriptional activities of pathways and miRNA

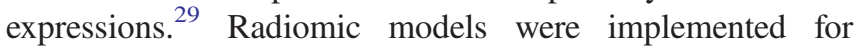
identification of Epithelial Growth Factor Receptor (EGFR) mutant status from $\mathrm{CT}$ through multiple logistic regression and pairwise selection ${ }^{30}$ and to decode ALK (anaplastic lymphoma kinase), ROS1 (c-ros oncogene 1), or RET (rearranged during transfection) fusions in lung adenocarcinoma. $^{31}$

Triple-negative breast cancer (TNBC) is likely to be identified by considering heterogeneity of background parenchymal enhancement, characterized by quantitative texture features on DCE-MRI, adds value to such differentiation models as they are strongly associated with the TNBC subtype. ${ }^{32}$ Furthermore, TNBC has been proven to be differentiated from fibroadenoma using ultrasound (US) radiomics. A radiomics score obtained by penalized logistic regression with a least absolute shrinkage and selection operator (LASSO) analysis showed significant difference between fibroadenoma and TNBC. ${ }^{33}$ The extraction of radiomic features from MR of GBM was able to predict immunohistochemically identified protein expression patterns. ${ }^{34}$

Despite large evidence of association among radiomics and genomics, few preclinical studies have demonstrated causal relationship between tumor genotype and radiomic. In one study, HCT116 colorectal carcinoma cells were grown as xenografts in the flanks of NMRI-nu mice. Then overexpression of GADD34 gene was induced by administration of HCT116 doxycycline (dox), or placebo was given. The radiomic analysis demonstrated that that gene overexpression causes change in radiomic features, as many features differed significantly between the dox-treated and placebo groups. ${ }^{4}$

\section{A.5. Clinical and macroscopic variables}

Radiomic features, derived from T2-w and ADC MRI scan, correlate with clinical variables that are relevant for patient's prognosis. These include prostate specific antigen (PSA) level ${ }^{35}$ in patients with prostate cancer, and Human Papilloma Virus (HPV) Status in head and neck squamous cell carcinoma. ${ }^{36,37}$ Given the well-known behavior of HPVpositive head and neck cancer which is likely to respond at a lower dose of chemoradiation, this opens the way to a CTbased patient stratification for a dose de-escalation.

\section{B. Radiomics in therapy}

Because radiomic features can describe histology ${ }^{22}$ and genetic footprint ${ }^{29-31}$ of the tumor, which are correlated with the tumor aggressiveness, they can be used to build models to predict the outcome, in terms of local/distant control or survival, of cancer therapy performed with various therapeutic options (radio-, chemotherapy, targeted molecular therapy, immunotherapy, nonionizing radiation) or a combination of them.

\section{B.1. Local control, response, and recurrence}

Radiomics predicts response to neoadjuvant chemoradiation assessed at time of surgery for nonsmall cell lung cancer (NSCLC) and locally advanced rectal cancer. ${ }^{38}$ Local control in patients treated with stereotactic radiotherapy for lung cancer was described using a PET and CT signature developed by using supervised principal component analysis was developed using features from PET and $\mathrm{CT}^{39} \mathrm{~A}$ radiomic model was developed using first-order statistics, GLCM, and geometrical measurements computed in T2-w and ADC 3T MRI by RF approach for biochemical recurrence of prostate cancer after radiotherapy. ${ }^{35} \mathrm{~A}$ total of 126 radiomic features were extracted using GLCM, GLGCM, Gabor transform, and GLSZM from contrast-enhanced 3T MRI using T1-w, T2$\mathrm{w}$, and DWI sequences to predict the therapeutic response of nasopharyngeal carcinoma (NPC) to chemoradiotherapy. ${ }^{40}$ Deep learning methods with radiomics are also proposed to predict outcomes after $\operatorname{liver}^{41}$ and lung cancers radiotherapy.

\section{B.2. Distant metastases}

Radiomic models to predict the development of distant metastases (DM) from NSCLC on patients treated with Stereotactic Body Radiotherapy (SBRT) patients for lung 
cancer were developed using features from $\mathrm{CT}^{42}$ or from PET-CT. ${ }^{39}$ Vallières et al. used texture-based model for the early evaluation of lung metastasis risk in soft-tissue sarcomas $^{43}$ from pretreatment FDG-PET and MRI scans comprising T1-w and T2-w fat-suppressed sequences (T2FS). A radiomic signature was developed to predict DM after locally advanced adenocarcinoma. ${ }^{44}$ Analysis of the peritumoral space can provide valuable information regarding the risk of distant failure, as more invasive tumors may have different morphologic patterns in the tumor periphery. An SVM classifier was trained to predict distant failure from radiomics analysis of the peritumoral space. ${ }^{45}$

\section{B.3. Survival}

Aerts et al. $^{3}$ built a radiomic signature consisting of a combination of four features in a retrospective lung cancer cohort, which was predictive for survival in head and neck and NSCLC independent cohorts. One textural feature calculated from GLCM, SumMean, ${ }^{46}$ was identified using the LASSO procedure as an independent predictor of overall survival that complements metabolic tumor volume (MTV) in decision tree. ${ }^{47}$ A radiomic signature was built from PET-CT for survival after SBRT for lung cancer. ${ }^{39}$ Deep learning was also proposed to stratify NSCLC patients according to mortality risk using standard of care $\mathrm{CT}^{48}$

\section{B.4. Molecular targeted therapy}

Many tumors commonly overexpress oncogenes such as the EGFR and respond to molecular targeted therapies such as EGFR tyrosine kinase inhibitor. From the change in features between the $\mathrm{CT}$ acquisitions before and 3 weeks after therapy it was possible to identify NSCLC patients responding to treatment with gefitinib. ${ }^{49} \mathrm{~A}$ radiomic prediction model was designed to stratify patients according to progression-free and overall survival after therapy with antiangiogenic for GBM. ${ }^{50}$

\section{B.5. Immunotherapy}

Cancer immunotherapy by immune checkpoint blockade is a promising treatment modality that is currently under strong development, and there is a great need for models to select patients responding to immunotherapy. In a retrospective multicohort study, an eight-feature radiomic signature predictive of the presence of CD8 T cells, which is related to the tumor-immune phenotype, was developed from CE-CT images, using elastic-net regularized regression method. ${ }^{51}$ The signature was successfully validated on external cohorts for discrimination of immune phenotype, and for the prediction of survival and response to anti-PD-1 or PD-L1 immunotherapy.

\section{B.6. Delta-radiomics}

The longitudinal study of features and of their change during the treatment, with the goal of predicting response to therapy, is called delta-radiomics. Features calculated from pretreatment and weekly intra-treatment $\mathrm{CT}$ change significantly during radiation therapy (RT) for NSCLC. ${ }^{52}$ Delta radiomics could possibly be performed by the Cone Beam CT (CBCT) devices for image guidance of radiotherapy treatment, thus allowing large-scale study of tumor response to total dose, fractionation and fraction dose. It has been shown that reproducible features can be extracted from $\mathrm{CBCT}^{53}$ predictive for overall survival in NSCLC patients as much as features from CT. ${ }^{54}$ Nevertheless, the studies on CBCT deltaradiomics are still limited to assessment of feasibility and reproducibility. ${ }^{55}$

\section{B.7. Prediction of side effects}

Radiomics-based models can help early identify the development of side effects such as radiation induced lung injury (RILI). The change from pre- to posttreatment (at 3, 6 , and 9 months) CT features significantly correlates with lung injury as scored by oncologist post-SBRT for lung cancer and was found to be correlated with dose and fractionation. ${ }^{56}$

A logistic regression-based classifier was constructed to combine information from multiple features to identify patients that will develop grade $\geq 2$ radiation pneumonitis among those who received RT for esophageal cancer. ${ }^{57}$ The addition of normal lung image features produced superior model performance with respect to traditional dosimetric and clinical predictors of radiation pneumonitis (RP), suggesting that pretreatment CT radiomic features should be considered in the context of RP prediction. CT radiomic features were extracted from the total lung volume defined using the treatment-planning scan for RP. ${ }^{58}$

\section{B.8. Differentiation of recurrence from benign changes}

The differentiation of tumor recurrence from benign radiation-induced changes in follow-up images can be a major challenge for the clinician. A radiomic signature consisting of five image-appearance features from CT demonstrated high discriminative capability to differentiate recurrence of lung tumor from consolidation and opacities in SBRT patients. ${ }^{59}$ Similarly, a combination of five radiomic features from CE-T1w and T2w MR were found to be capable of distinguishing necrosis from progression in follow-up MR images in patients treated with Gamma Knife radiosurgery for brain metastases. ${ }^{60}$

\section{B.9. Nonionizing radiation and other therapies}

Radiomic features in MRI respond differently when Laser interstitial thermal therapy (LITT), a highly promising focal strategy for low-grade, organ-confined prostate cancer, is performed on cancer or healthy prostate tissue. A radiomic signature then could allow to assess if prostate cancer is successfully ablated. ${ }^{61}$ A radiomic model was predictive of complete response after transcatheter arterial chemoembolisation 
combined with high-intensity focused ultrasound treatment in hepatocellular carcinoma. $^{62}$

\section{RADIOMICS ANALYSIS WITH MACHINE AND DEEP LEARNING METHODS}

\section{A. Preprocessing}

Prior to radiomics analysis, preprocessing steps need to be applied to the images, which aim at reducing image noise, enhancing image quality, enabling the reproducible and comparable radiomic analysis. For some imaging modalities, such as PET, the images should be converted to a more meaningful representation (standardized uptake value, SUV). Image smoothing can be achieved by average or Gaussian filters. ${ }^{63}$ Voxel size resampling is important for datasets that have variable voxel size. ${ }^{64}$ Specifically, isotropic voxel size is required for some texture feature extraction. There are two main categories of interpolation algorithms: Polynomial and spline interpolation. Nearest neighbor is a zero-order polynomial method that assigns gray-level values of the nearest neighbor to the interpolated point. Bilinear or trilinear interpolation and bicubic or tricubic interpolation are often used for two-dimensional (2D) in-plane interpolation or 3D cases. Cubic spline and convolution interpolation are third order polynomial method that interpolates smoother surface than linear method, while being slower in implementation. Linear interpolation is a rather commonly used algorithm, since it neither leads to the rough blocking artifacts images that are generated by nearest neighbors, nor will it cause out-ofrange gray levels that might be produced by higher order interpolation. $^{65}$

In the context of feature-based radiomics analysis, as discussed below, the computation of textures would require discretization of the gray levels (intensity values). There are two ways to do the discretization: fixed bin number $\mathrm{N}$ and fixed bin width B. For fixed bin number, we first decide a fixed number of $\mathrm{N}$ bins, and the gray levels will be discretized into these bins using the formula below:

$$
X_{d, k}=\left\{\begin{array}{ll}
{\left[N_{g} \frac{X_{g l, k}-X_{g l, \text { min }}}{X_{g l, m a x}-X_{g l, \text { min }}}\right]+1} & X_{g l, k}<X_{g l, \text { max }} \\
N_{g} & X_{g l, k}=X_{g l, \text { max }}
\end{array},\right.
$$

where $X_{g l, k}$ is the intensity of $k$ th voxel.

For fixed bin width, starting at a minimum $X_{g l, m i n}$, a new bin will be assigned for every intensity interval of $w_{b}$. Discretized gray levels are calculated as follow:

$$
X_{d, k}=\left[\frac{X_{g l, k}-X_{g l, m i n}}{w_{b}}\right]+1 .
$$

The fixed bin number method is better when the modality used is not well calibrated. It maintains the contrast and makes the images of different patients comparable, but loses the relationship between image intensity, while fixed bin size method keeps the direct relationship with the original scale. Some investigations about the effect of both methods have shown that fixed bin size method offered better repeatability and thus may be suitable for intra- and inter-patient studies, however, this remains a subject of ongoing research. ${ }^{66,67}$ In $\mathrm{CT}$ radiomics the image pixel intensity maps to the Hounsfield Units (HU) and thus is much more directly comparable and interpretable. MRI-related modalities are more challenging since the pixel intensities are not directly interpretable, rather need to normalized relative to some standard reference (e.g., contralateral brain, or normal appearing white matter in neuroimaging, psoas muscle in abdominal imaging, etc.).

\section{B. Machine and deep learning algorithms for radiomics}

Machine and deep learning algorithms provide powerful modeling tools to mine the huge amount of image data available, reveal underlying complex biological mechanisms, and make personalized precision cancer diagnosis and treatment planning possible. Hereafter, two main types - feature-engineered (conventional radiomics) and nonengineered (deep learning based) radiomics modeling methods — will be briefly introduced. Generally speaking, machine learning methods can also be divided into supervised, unsupervised and semisupervised for both feature-based and featureless methods. Each of these categories will be briefly discussed in the following sections. A workflow diagram illustrating the radiomics analysis process after image acquisition is shown in Fig. 1.

\section{B.1. Feature-engineered radiomics methods}

Traditionally, the radiomic features being extracted are hand-crafted features that capture characteristic patterns in the imaging data, including shape-based, first-, second-, and higher order statistical determinants and model-based (e.g., fractal) features. Feature-based methods require a segmentation of the region of interest (ROI), either through a manual, semiautomated, or automatic methods. Shape-based features are external representations of a region that characterize the shape, size, and surface information of the ROIs. ${ }^{68}$ Typical metrics include sphericity, and compactness. ${ }^{3,43,69,70}$ Firstorder features (e.g., mean, median) describe the overall intensity and variation of the ROIs, while ignoring spatial relations. ${ }^{8,24}$ Second-order (texture) features in contrast can provide inter-relationships among voxels. Textural features can be extracted from different matrices, for example, graylevel co-occurrence matrix (GLCM), gray-level run-length matrix (GLRLM), etc. ${ }^{35,46,71}$ Semantic features are another type of feature that can be extracted from medical images. These features describe qualitative features of the image typically used in the radiology workflow.

Hundreds or even thousands of radiomic features are not uncommon when we deal with outcome modeling. Feature selection and/or extraction thus is a crucial step that aims at obtaining the optimal feature subset or feature representation that correlates most with the endpoint and meanwhile correlates least between each other. After the feature subset is 


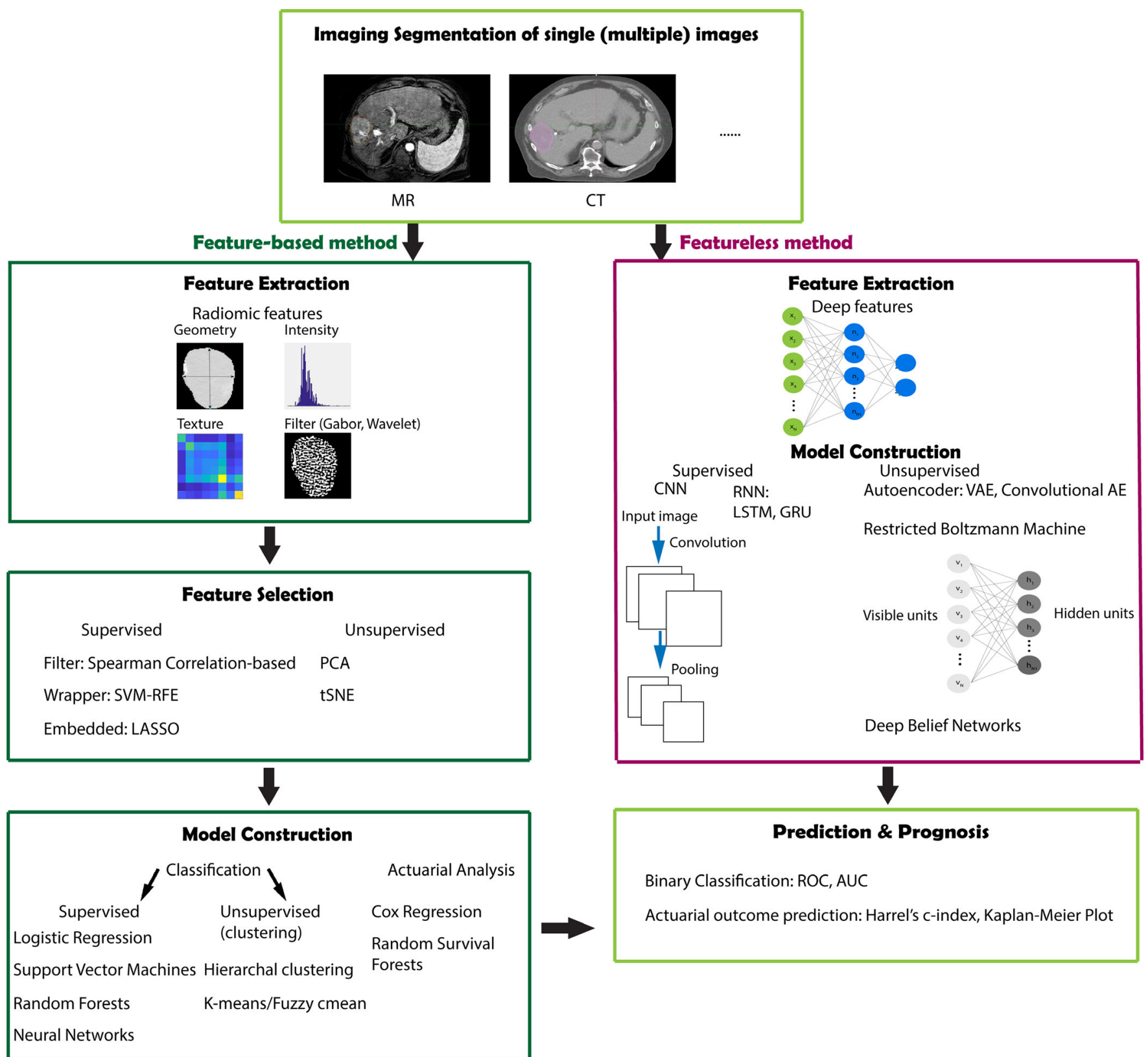

FIG. 1. Workflow for radiomics analysis with feature-based (conventional machine learning) and featureless (deep learning) approaches.

obtained, various machine learning algorithms can be applied based on them. Fourteen feature selection and 12 classification methods were evaluated in terms of their predictive performance on two independent lung cancer cohorts. ${ }^{72}$ Sometimes, the feature selection and model construction can be implemented together, called the embedded method, such as least absolute shrinkage and selection operator (LASSO). ${ }^{73}$ In contrast, wrapper methods select the features based on the models' performance for different subsets of features, for which we need to rebuild the model again after features are selected, for instance, recursive feature elimination support vector machines (SVM-RFE). Filter method also separates the feature selection and model construction processes, whose uniqueness of it is its independence of the classifier being used for the subsequent model building, such as Pearson correlation-based feature ranking. In any feature selection method, it is essential to ensure that there is no "double dipping" into the training data for both feature selection, hyperparameter optimization, and model selection. Rather the methods of "nested cross validation" should be used in order to prevent overfitting or incorrect estimates of generalization. According to whether or not the labels (ground truths) are used, feature selection and extraction can be divided into supervised, unsupervised and semisupervised ways. The three feature selection methods discussed above are mostly supervised. Examples of unsupervised methods are principle component analysis (PCA), ${ }^{74}$ clustering and $t$-distributed stochastic neighbor embedding ( $t$-SNE). ${ }^{75}$ PCA uses an 
orthogonal linear transformation to convert the data into a new coordinate system so that large variances are projected to orthogonal coordinates. Clustering is another feature extraction algorithm which aims at finding relevant features and combining them by their cluster centroids based on some similarity measure, such as K-means and hierarchical clustering. ${ }^{76}$ Unsupervised consensus clustering identified robust imaging subtypes using dynamic CE-MRI data for patients with breast cancer. ${ }^{77} t$-distributed stochastic neighbor embedding is a dimension reduction method capable of retaining the local structure (pairwise similarity) of data, while revealing some important global structure.

In the medical field, two types of questions are mainly investigated, binary problems (classification), such as whether or not a disease has recurred, the patient is alive beyond certain time threshold, etc; and survival analysis, that is able to show if a risk factor or treatment affects time to event. For the classification problem logistic regression fits the coefficients of the variables to predict a logit transformation of the probability of the presence of the event. SVM, frequently used in Computed Aided Diagnosis $(\mathrm{CAD})^{6}$ and radiomics, ${ }^{32,59,76,78}$ learns an optimal hyperplane that separates the classes as wide as possible, while trying to balance with misclassified cases. SVM can also perform non-linear classification using the "kernel trick" - different basis functions (e.g., radial basis function), mapping to higher dimensional feature space. The hyperplane maximizes the margin between the two classes in a nonlinear feature space. SVM also tolerates some points on the wrong side of the boundary, thus improving model robustness and generalization. ${ }^{79} \mathrm{RF}$ is based on decision trees, a popular concept in machine learning especially in the field of medicine, because their representation of hypotheses as sequential "ifthen" resembles human reasoning. ${ }^{80} \mathrm{RF}$ applies bootstrap aggregating to decision trees and improve the performance by lowering the high variance of the trees. ${ }^{81}$ Risk assessment models (classification and survival) were constructed via RFs and imbalance adjustment strategies for locoregional recurrences and distant metastases in head and neck cancer. $^{82}$

Neural networks, though usually used in the featureless context, can also be used in conventional feature selection and modeling. ${ }^{22,38,78}$ These algorithms are mainly for supervised learning, while in particular in the medical field, there are a lot of data without labeling, in these cases, semisupervised learning can be applied to make use of the unlabeled data combined with the small amount of labeled data. The self-training is bootstrapped with additional labeled data obtained from its predictions. ${ }^{83}$ The transductive SVM (TSVM) tries to keep the unlabeled data as far away from the margin as possible. ${ }^{84}$ Graph-based methods construct a graph connecting similar observations and enable the class information being transported through the graph. ${ }^{85}$

For the survival analysis, Cox regression, ${ }^{86}$ random survival forests, ${ }^{87}$ and support vector survival ${ }^{88}$ methods are also available to investigate the presence of a set of variables that may affect survival time. Due to the length limit, we will not go into the details. Interested readers can refer to the references to read more about these algorithms.

\section{B.2. Non feature-engineered radiomics methods}

Although hand-crafted features introduced above provide prior knowledge, they also suffer from the tedious designing process and may not faithfully capture the underlying imaging information. Alternatively, with the development of deep learning technologies based on multilayer neural networks, especially the convolutional neural networks $(\mathrm{CNN})$, the extraction of machine learnt features is becoming widely applicable recently. In deep learning, the processes of data representation and prediction (e.g., classification or regression) are performed jointly. ${ }^{89}$ In such a case, multistack neural layers of varying modules (e.g., convolution or pooling) with linear/nonlinear activation functions perform the task of learning the representations of data with multiple levels of abstraction and subsequent fully connected layers are tasked with classification, for instance. A typical scenario to get such features is to use the data representation CNN layers as feature extractor. Each hidden layer module within the network transforms the representation at one level. For example, the first level may represent edges in an image oriented in a particular direction, the second may detect motifs in the observed edges, the third could recognize objects from ensembles of motifs. ${ }^{89}$ Patch-/pixel-based machine learning (PML) methods use pixel/voxel values in images directly instead of features calculated from segmented objects as in other approaches. ${ }^{89,90}$ Thus PML removes the need for segmentation, one of the major sources of variability of radiomic features. Moreover, the data representation removes the feature selection portion eliminating associated statistical bias in the process. For the CNN network, either self-designed (from scratch) or existing structures, for example, VGG, ${ }^{91}$ Resnet, ${ }^{92}$ can be used. Depending on the data size, we can choose to fix the parameters or fine-tune the network using our data, also called transfer learning. Instead of using deep networks as feature extractors, we can use them directly for the whole modeling process. Similarly to the conventional machine learning methods, there are also supervised, unsupervised, and semi-supervised methods. CNN are similar to regular neural networks, but the architecture is modified to fit to the specific input of large-scale images. Inspired by the Hubel and Wiesel's work on the animal visual cortex, ${ }^{93}$ local filters are used to slide over the input space in CNNs, which not only exploit the strong local correlation in natural images but also reduce the number of weights significantly by sharing weights for each filter. Recurrent neural networks (RNN) can use their internal memory to process sequence inputs and take the previous output as inputs. There are two popular types of RNN - Long short-term memory (LSTM) ${ }^{94}$ and Gated recurrent units (GRU). ${ }^{95}$ They were invented to solve the problem of vanishing gradient for long sequences by internal gates that are able to learn which data in the sequence is important to keep or discard. Deep autoencoders (AE), which are unsupervised learning algorithms, have been 
applied to medical imaging for latent representative feature extraction. There are variations to the AEs, such as variational autoencoders that resemble the original $\mathrm{AE}$ and variational Bayesian methods to learn a probability distribution that represents the data, ${ }^{96}$ convolutional autoencoders that preserve spatial locality, ${ }^{97}$ etc. Another unsupervised method is the restricted Boltzmann machine (RBM), which is consists of visible and hidden layers. ${ }^{98}$ The forward pass learns the probability of activations given the inputs, while the backward pass tries to estimate the probability of inputs given activations. Thus, the RBMs lead to the joint probability distribution of inputs and activations. Deep belief networks can be regarded as a stack of RBMs, where each RBM communicates with previous and subsequent layers. RBMs are quite similar with AEs, however, instead of using deterministic units, like RELU, RBMs use stochastic units with certain distribution. As mentioned above, labeled data are limited, especially in the medical field. Neural network based semisupervised approaches combine unsupervised and supervised learning by training the supervised network with an additional loss component from the unsupervised generative models (e.g., AEs, RBMs). ${ }^{99}$

Machine learning methods are highly effective with large number of samples; however, they suffer from overfitting pitfalls with limited training samples. For deep learning, data augmentation (e.g., by affine transformation of the images) during training is commonly implemented. Transfer learning is another way to reduce the difficulty in training. Using deep models trained on other dataset (natural images) and then fine-tune on the target dataset. The structures of the networks can also be modified to reduce overfitting, such as by adding dropout and batch normalization layers. Dropout randomly deactivates a fraction of the units during training and can be viewed as a regularization technique that adds noise to the hidden units. ${ }^{100}$ Batch normalization reduces the internal covariate shift by normalizing for each training mini-batch. ${ }^{101}$

Comparing with feature-based methods, deep learning methods are more flexible and can be used with some modifications in various tasks. In addition to classification, segmentation, registration, and lesion detection are widely explored by deep learning techniques. Fully CNN (FCN), trained end-to-end, merge features learnt from different stages in the encoders and then upsampling low-resolution feature maps by deconvolutions. ${ }^{102}$ U-Net, built upon FCN, with the pooling layers being replaced by upsampling layers, resulted in a nearly symmetric U-shaped network. $^{103}$ Skipping structures combines the context information with the unsampled feature maps to achieve higher resolution. CNN, trained end-to-end from clinical images were directly used for binary classification of skin cancer and achieved performance on par with experts. ${ }^{104}$ Chang et al. proposed a multiscale convolutional sparse coding method that provides an unsupervised solution for learning transferable base knowledge and fine-tuning it towards target tasks. ${ }^{105}$

\section{C. Validation and benchmarking of radiomics models}

Once models are developed using the selected predictors, quantifying the predictive ability of the models (validation) is necessary. Based on the TRIPOD criteria, ${ }^{106}$ there are four types of validation: (1a) developing and validating on the same data, which gives apparent performance. This evaluation is usually optimistic estimation of the true performance. (1b) Developing the models using all the data, then using resampling techniques to evaluate the performance. (2a) Randomly split the data into two groups for development and validation separately. (2b) Split the data nonrandomly (e.g., by location or time), which is stronger than (2a), (3), and (4). Develop the model using one data set and validate on separate data. It is ideal if there is a separate data set for external validation, however, in the frequent case that only a single data set is available, internal validation (1b) is required. Two popular resampling methods are bootstrapping and cross-validation. Feature selection, which is required before machine learning, should precede cross-validation, or it will lead to a selection bias due to the leak of information by the prefiltering of the features. ${ }^{107}$

Radiomic classifiers output a score that indicates the likelihood of one event to happen, and a threshold, to generate positive or negative predictions according to the task at hand. For example, fewer false positives would be required if we are implementing a conservative experiment, thus larger threshold will be preferred. Classifiers are evaluated using either a numeric metric (e.g., accuracy), or the so-called confusion matrix, or a graphical representation of performance, such as a receiver operating characteristic curve (ROC), a 2D graph with true positive rate being the $\mathrm{Y}$ axis, and false positive rate the $\mathrm{X}$ axis. It has the advantage that they show classifier performance without regard to threshold and class distribution, thus widely used in model evaluation. The area under an ROC curve (AUC) is more convenient when comparing, and is equivalent to the probability that the classifier will rank a randomly chosen positive instance higher than a randomly chosen negative instance. ${ }^{108}$ For survival analysis, Harrell's C index ${ }^{109}$ is commonly used to measure discrimination ability of the model, which is motivated by Kendall's tau correlation. Harrell defines the overall $\mathrm{C}$ index as the proportion of all usable pairs in which the predicted risk probabilities and outcomes are concordant (Usable pairs are two cases that at least one of them is event). ${ }^{110}$

Kaplan-Meier (KM) curves are used to estimate the survival function from lifetime data, and also used to compare different risk groups. The risk groups can be patients that are treated with certain plan and the control group, or they can be the outputs from a survival model (e.g., Cox model) that divides the patients into high- and low-risk groups. It is highly recommended to visualize confidence intervals of the curves. The log rank test gives a quantitative evaluation of the statistical significance of the difference for different curves, which is also widely provided for KM curves. ${ }^{111}$ 


\section{IMPLEMENTATION IN MEDICAL PHYSICS PRACTICE}

\section{A. Software tools for radiomics}

In most published research studies in radiomics, in-house developed methods are used. However, some research groups developed image analysis/radiomic software tools, both commercial and open source, available to the scientific community. The main goals of these tools are: (a) to speed up the development of competences based on more recent skills on radiomics; (b) to allow reproducibility and comparability of results from different research groups, and (c) to standardize both feature definitions and computation methods to guarantee the reliability of radiomic results. ${ }^{112,113}$

Table I shows a list of the software, web platforms, and toolkits available free of charge for the extraction of radiomics features, along with some of their main functionalities and relevant information. Given the high pace of radiomic developments, the list is not exhaustive and does not intend to cover all possible solutions. Furthermore, considering recent and increased interest in the radiomic field, many other dedicated tools are under development. All the open-source solutions shown in this overview have been implemented by research teams (MaZda, ${ }^{114}$ LifeX, ${ }^{115}$ ePAD ${ }^{116}$ HeterogeneityCAD, ${ }^{3}$ PyRadiomics/Radiomics, ${ }^{117}$ QuantImage, ${ }^{118}$ the Texture Analysis Toolbox, ${ }^{43}$ QIFE, ${ }^{119}$ IBEX, ${ }^{120}$ and MedomicsLab) and are capable of analyzing CT, MRI, and PET, some of them can process also other medical images, such as mammography, radiography, or ultrasound.

Four software programs (MaZda, LifeX, ePAD, IBEX) offer the possibility of manually or automatically segmenting medical images. Three toolkits (HeterogeneityCAD, PyRadiomics/Radiomics, QIFE) are designed exclusively for the extraction of features. They can be embedded in more complete solutions (e.g., 3D Slicer ${ }^{121}$ ). Morphological, first, second, and third order statistical features can be extracted by all software solutions, except for ePAD. Four of them (TexRAD, MaZda, PyRadiomics/Radiomics, IBEX) offer also the possibility of extracting features from filtered images. Of note, MEDomicsLab is an open-source software currently being developed by a consortium of research institutions, which will be available in the second half of 2019 .

\section{B. Commercial programs for radiomics}

Commercial software programs are also becoming increasingly available due to the interest of many medical device incumbents as well as newcomers such as commercial spin-off of research groups or de novo start-up companies. Such software programs can be divided into:

\section{RESEARCH PLATFORMS}

Research platforms enable the discovery of new signatures by linking quantitative imaging biomarkers, clinical and omics data to clinical endpoints. They are usually considered nonmedical devices in that they do not affect the clinical routine, run usually on independent workstations, and are not used to drive clinical decisions. Their main differentiator from open access software consists of workflow optimization and efficiency improvements, enabling an automatic, end-toend seamless processing pipeline. TexRad ${ }^{\circledR}$, QIDS $\AA$, Radio$\mathrm{miX}$, iBiopsy ${ }^{\circledR}$, and EVIDENS offer research capabilities at a different level, ranging from simple features extraction to image filter application and machine learning modules. In the research mode, these software programs are usually open to process any 3D image, DICOM or not, up to 2D digital pathology images (histomics or pathomics).

\section{A. Clinically validated software programs}

In order to use decision support systems (DSSs), based on an already discovered signatures and thoroughly validated on large independent datasets, also known as clinical grade DSS, in clinical practice, a regulatory clearance is usually needed, as they fall within the definition of medical devices in many regulatory systems, for example, class I or II medical device as a function of their intended use (e.g., mere support to decision vs a computer-aided diagnosis/prognosis). DSSs are usually limited to a specific modality, mostly $\mathrm{CT}$, and to a specific disease in a specific body district: these constraints come primarily from the intended use definition to which these DSSs are subjected to be compliantly marketed.

Research tools or clinical grade DSSs can be embedded into more comprehensive platforms such as Picture Archive and Communication Systems (PACS), Hospital Information Systems (HIS), Oncology Information Systems (OIS) or Treatment Planning Systems (TPS), or being stand-alone. Usually, large medical device incumbents tend to embed DSSs into their research or clinical solutions, while newcomers often offer their solution as a standalone system.

It is not unusual that large medical device players embed open access or commercial software programs to provide their customers with the possibility of exploring or exploiting radiomic potential: examples are IntelliSPace Discovery (Philips, the Netherlands) which interfaces to Pyradiomics, Advantage Workstation (GE, Buc, France) which interfaces through a plugin to Quantib ${ }^{\mathrm{TM}}$ Brain or Syngo.via Frontier (Siemens, Erlangen, Germany) which interfaces to RadiomiX. It is also beneficial to mention the platform (www.en voyai.com) which offers the possibility of sharing applications and, once solutions reached the product maturity, to commercialize them.

\section{CURRENT CHALLENGES AND RECOMMENDATIONS}

\section{A. Interpretability issues}

It is recognized that machine learning algorithms tend to generally trade interpretability for better prediction. Hence, clinicians are still reluctant to embrace these methods as part 


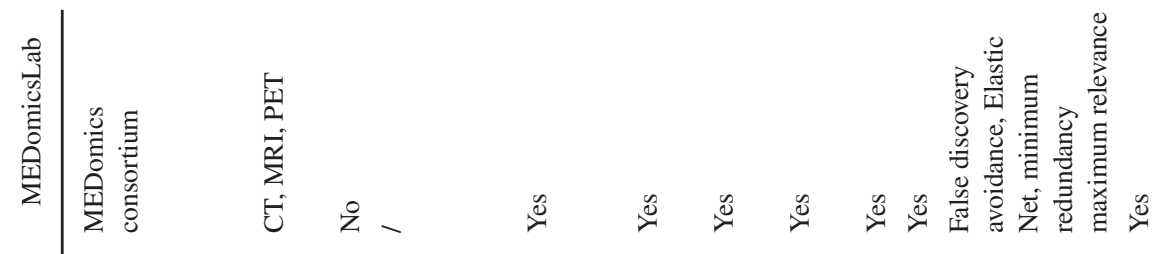

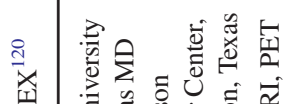

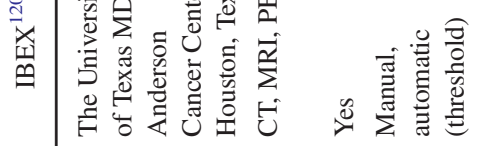

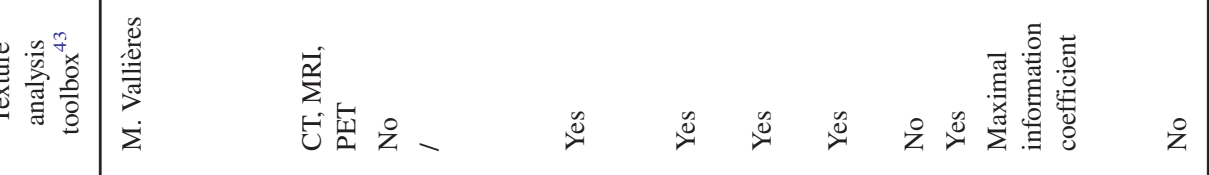

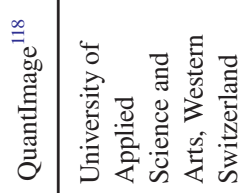

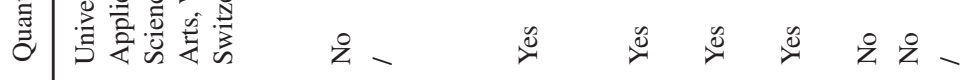

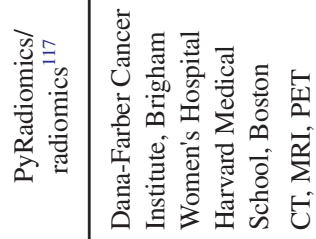

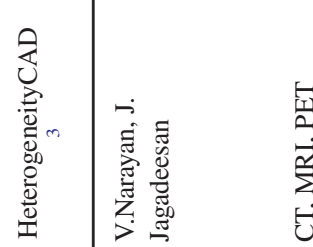

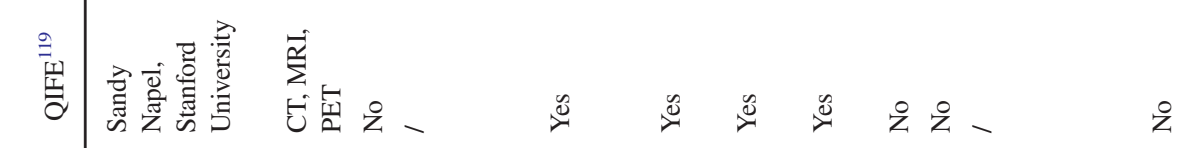

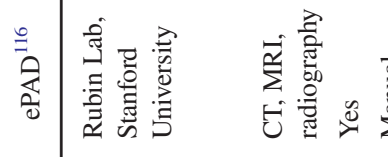

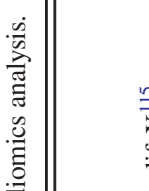

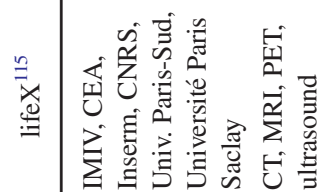

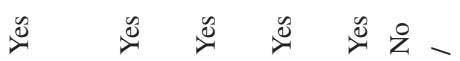

そ̊ำ

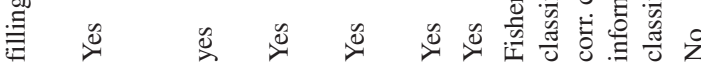


of their clinical practice, because they have long been perceived them as "black boxe," meaning that it is difficult to determine how they arrive at their predictions. For example, it is difficult to understand deep neural networks due to the large number of interacting, nonlinear parts. ${ }^{122,123}$ In order to improve interpretability of radiomics for the clinician, methods based on graph approaches can be utilized, ${ }^{124}$ and in the context of deep learning better visualization tools are being developed such as maps highlighting regions of the tumor that impact the prediction of the deep learning classifier are also being proposed. ${ }^{123}$

\section{B. Repeatability and reproducibility issues}

In radiomics, repeatability is measured by extraction of features from repeated acquisition of images under identical or near-identical conditions and acquisition parameters, ${ }^{125}$ whereas reproducibility or robustness, is assessed when measuring system or parameters differ. These can be assessed by use of digital or physical phantoms. Physical phantoms usually contain inserts of different with different density, shape, or texture properties in order to produce a wide range of radiomics feature values. These phantoms allow to assess the reproducibility or robustness of the entire workflow, from image acquisition to extraction of radiomic features. Their major drawback is that they do not reflect the variability of human anatomy in the clinical scenario.

A phantom for radiomics was created for use with $\mathrm{CT}^{113}$ or $\mathrm{CBCT}^{126}$ called Credence Cartridge Radiomics (CCR) Phantom. This consisted of ten cartridges with different density and texture properties in order to produce a wide range of radiomics feature values: wood, rubber, cork, acrylic, and plaster. Phantoms for PET with heterogeneous lesions have been also proposed, for example, with different 3D printed inserts reflecting different heterogeneities in FDG uptake. ${ }^{127}$

Digital phantoms are usually scans of patients acquired under controlled conditions. They are therefore realistic, but cannot be used for studying radiomic features' sensitivity to the image acquisition and its parameters. A dataset consisting of 31 sets of repeated CT scans acquired approximately 15 min apart is now publicly accessible through The Reference Image Database to Evaluate Therapy Response (RIDER). This dataset allows "test-retest" analysis, a comparison of the results from images acquired within a short time on the same patient. ${ }^{128}$

\section{C. Factors affecting stability}

For CT, inter-scanner variability of image features produces differences in extracted features that are comparable to the variability in patient images acquired by the same scanner. ${ }^{113}$ The choice of methods of reconstruction, such as filtered back projection or iterative algorithm, also affect radiomic feature. ${ }^{129}$ Smoothing of the image and reducing the slice thicknesses can improve reproducibility of CT-extracted features. ${ }^{128,130}$ In PET imaging, textural features are sensitive to different acquisition modes, ${ }^{131,132}$ reconstruction algorithms, and their user-defined parameters such as the number of iterations, the postfiltering level, input data noise, matrix size, and discretization bin size. ${ }^{133,134}$

Radiomic features extracted from MRI scans depend on the field of view, field strength, reconstruction algorithm, and slice thickness. Results of the DCE-MRI depend on the contrast agent dose, method of administration, and the pulse sequence used. The radiomic features extracted from DWMRI depend on acquisition parameters and conditions as kspace trajectory, gradient strengths and b-values. The repeatability of MR-based radiomic features has been investigated ${ }^{135}$ using a ground truth digital phantom of brain glioma patients and an MRI simulator capable of generating images according to different acquisition (field strength, pulse sequence, arrangement of field coils) and reconstruction methods. It was found that some features are subject to small changes, compared with clinical effect size.

In presence of significant respiratory tumor motion as in the case of lung cancer, conventional PET images are influenced by motion as, because of their relatively long acquisition times, the counts measured are averaged over multiple breathing cycles. Respiratory-gated PET accounts for respiratory motion and textural features from gated PET have been found robust. ${ }^{136}$

Segmentation affects the radiomics workflow, regardless of the imaging technique, because many extracted features depend on the segmented region. ${ }^{2,5}$ Semiautomatic segmentation algorithms may improve the stability of radiomic features, ${ }^{137}$ and recently available fully automatic segmentation tools may be as accurate as manual segmentation by medical experts. $^{138}$

The studies on the comparisons of the performance of many classifier and feature selection methods indicate that the choice of classification method is the most dominant source of models' predictive performance variability. ${ }^{72}$ Fourteen feature selection algorithms were compared on a set of 464 lung cancer patients considering 440 radiomic variables. ${ }^{76}$ The feature selection method based on the Wilcoxon signed-rank (WLCX) test had the highest prognostic performance with high stability against data perturbation. Interestingly, WLCX is a simple univariate method based on ranks, which does not take into account the redundancy of selected features during feature ranking. In a comparison of performance of 24 feature selection methods for radiomic signature building for lung cancer histology it was shown that RELIEF with its variants were the best performing methods. ${ }^{22}$

\section{D. Quality, radiomics quality score}

The workflow for radiomic studies involves several steps, from data acquisition, selection, and curation, to feature extraction, feature selection, and modeling. There is 


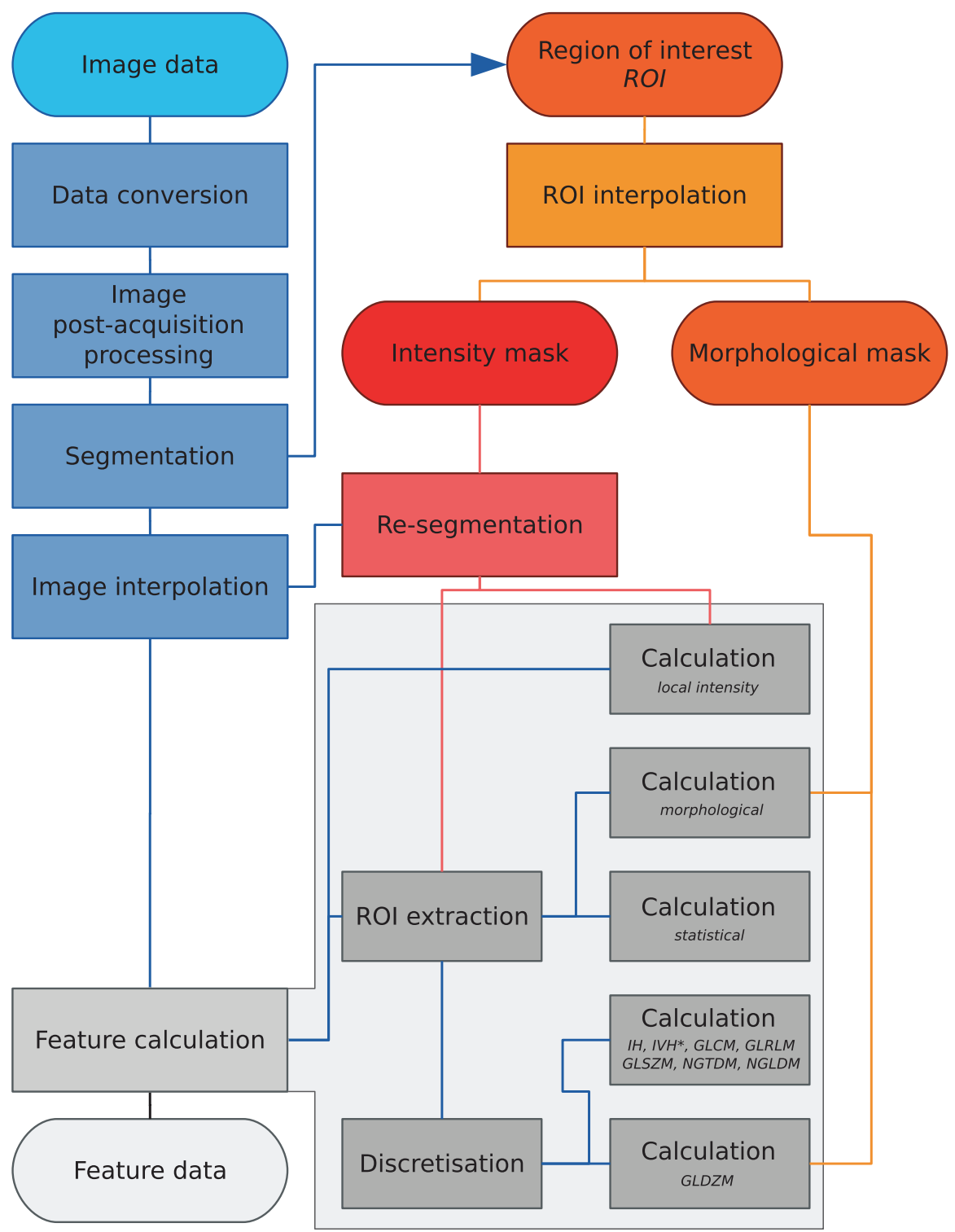

FIG. 2. Radiomics computation workflow as defined by the image biomarker standardization initiative.

an important need that radiomics studies are properly designed and reported to ensure the field can continue to develop and produce clinically useful tools and techniques. A number of issues can arise providing misleading information, including imaging artifacts, poor study design, overfitting of data, and incomplete reporting of results. ${ }^{8,139}$ Although imaging artifacts are inevitable in medical imaging, consistent imaging parameters may help reduce variability in radiomic features. ${ }^{126}$ To minimize the potential of overfitting of radiomic models, ten patients are needed for each feature in the final model. ${ }^{140}$ Ideally, an independent external validation dataset is also used to confirm the prognostic ability of any radiomic model. The radiomics quality score (RQS) has recently been developed to assess all areas of a radiomic study and determine whether it is compliant with best practice procedures, ${ }^{139}$ emulated from the TRIPOD initiative previously described.

\section{E. Standardization and harmonization}

Although research in the field of radiomics has drastically increased over the past several years, there still remains a lack of reproducibility and validation of current radiomic models. There are currently no guidelines and standard definitions for radiomic features and for constructing these features into clinical models. Current initiatives are underway to improve standardization and harmonization in radiomic studies.

As a part of radiomic signature validation, there are efforts to explore distributed feature sharing and model development across contributing institutions. ${ }^{141} \mathrm{~A}$ key component in this exercise is the assessment and redressal of batch effects ${ }^{142}$ and confounding variables across contributing sites, so as to ameliorate systematic yet unmeasured sources of variation. Another key component is the use of methods to harmonize data as well as model parameters across study sites, with the 
TABLE II. Reporting guidelines on the computation of radiomics features (adapted from Refs. [65] and [146]).

General

Image acquisition

Volumetric analysis

Workflow structure

Software

Image preprocessing

Conversion

Processing

ROI segmentation ${ }^{\mathrm{a}, \mathrm{b}}$

Interpolation

Voxel dimensions

Image interpolation method

Intensity rounding

ROI interpolation method

ROI partial volume

ROI re-segmentation

Inclusion/exclusion criteria

Image discretization

Discretization method

Discretization parameters

Feature calculation

Features set

Features parameters

Calibration

Image processing steps

Features calculation
Acquisition protocols and scanner parameters: equipment vendor, reconstruction algorithms and filters, field of view and acquisition matrix dimensions, MRI sequence parameters, PET acquisition time and injected dose, CT x-ray energy $(\mathrm{kVp})$, exposure (mAs), etc

Imaging volumes are analyzed as separate images (2D) or as fully connected volumes (3D)

Sequence of processing steps leading to the extraction of features

Software type and version of code used for the computation of features

How data were converted from input images: for example, conversion of PET activity counts to SUV, calculation of ADC maps from raw DW-MRI signal, etc

Image processing steps taken after image acquisition: for example, noise filtering, intensity nonuniformity correction in MRI, partial-volume effect corrections, etc

How regions of interests (ROIs) were delineated in the images: software and/or algorithms used, how many different persons and what expertise (specialty, experience), how a consensus was obtained if several persons carried out the segmentation, in automatic or semiautomatic mode, etc

Original and interpolated voxel dimensions

Method used to interpolate voxels values (e.g, linear, cubic, spline, etc.) as well as how original and interpolated grids were aligned

Rounding procedures for noninteger interpolated gray levels (if applicable), for example, rounding of Hounsfield units in CT imaging following interpolation

Method used to interpolate ROI masks. Definition of how original and interpolated grids were aligned

Minimum partial volume fraction required to include an interpolated ROI mask voxel in the interpolated ROI (if applicable): for example, a minimum partial volume fraction of 0.5 when using linear interpolation

Criteria for inclusion and/or exclusion of voxels from the ROI intensity mask (if applicable), for example, the exclusion of voxels with Hounsfield units values outside a predefined range inside the ROI intensity mask in CT imaging

Method used for discretizing image intensities prior to feature extraction: for example, fixed bin number, fixed bin width, histogram equalization, etc

Parameters used for image discretization: the number of bins, the bin width and minimal value of discretization range, etc

Description and formulas of all calculated features

Settings used for the calculation of features: voxel connectivity, with or without merging by slice, with or without merging directional texture matrices, etc

Specifying which image processing steps match the benchmarks of the IBSI

Specifying which feature calculations match the benchmarks of the IBSI

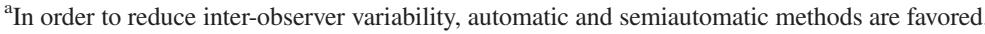

${ }^{\mathrm{b}}$ In multimodal applications (e.g., PET/CT, PET/MRI, etc.) ROI definition may involve the propagation of contours between modalities via co-registration. In that case, the technical details of the registration should also be provided.

intent of meaningful comparisons across clinical population. ${ }^{143}$ Such efforts are necessary to enable the widespread and generalizable development of models that are transportable across institutions. In addition to the careful calibration and stability analysis of radiomic features within predictive models, there is also a need for ensuring model robustness through approaches like noise injection. ${ }^{144}$ Adversarial training approaches from neural networks can have value in the modern deep learning modeling area by incorporating not only positive examples but negative ones too. ${ }^{145}$ The workflow for computing features is complex and involves many steps, often leading to incomplete reporting of methodological information (e.g., texture matrix design choices and gray-level discretization methods). As a consequence, few radiomics studies in the current literature can be reproduced from start to end.

To accelerate the translation of radiomics methods to the clinical environment, the image biomarker standardization initiative (IBSI) ${ }^{65}$ has the goal to provide standard definitions and nomenclature for radiomic features, reporting guidelines, and to provide benchmark datasets and values to verify image processing and radiomic feature calculations. Figure 2 presents the standardized radiomics workflow defined by the IBSI. The IBSI aims at standardizing both the computation of features and the image processing steps required before feature extraction. For this purpose, a simple digital phantom was designed and used in Phase 1 of the IBSI to standardize the computation of 172 features from 11 categories. In Phase 
TABLE III. Quality factors in radiomics studies (adapted from Refs. [139] and [146]).

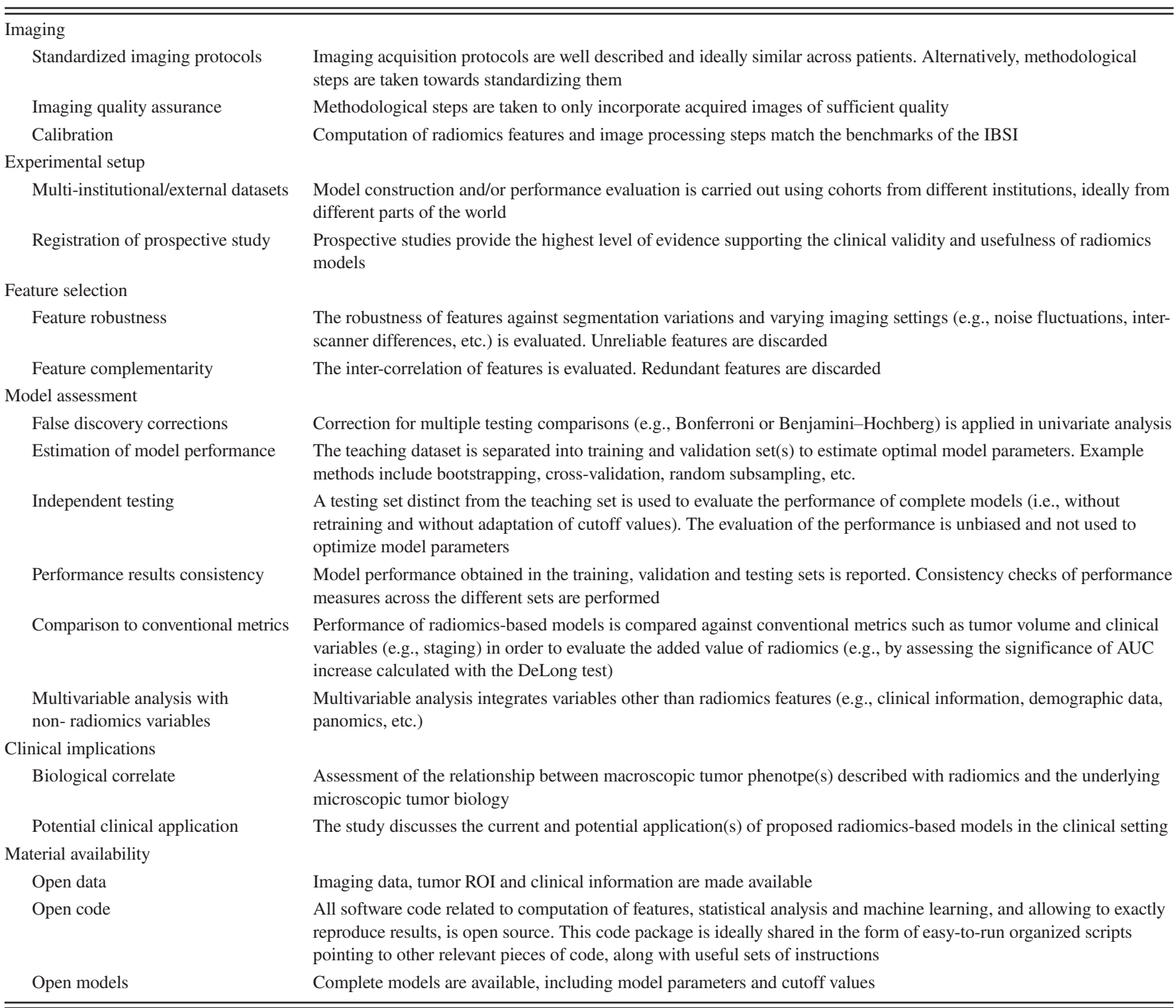

2 of the IBSI, a set of CT images from a lung cancer patient was used to standardize radiomics image processing steps using five different combinations of parameters including volumetric approaches (2D vs 3D), image interpolation, resegmentation and discretization methods. The initiative is now reaching completion and a consensus on image processing and computation of features has been reached over time.

Overall, the use of standardized computation methods would greatly enhance the reproducibility of radiomics studies, and it may also lead to standardized software solutions available to the community. It would also be desirable that the code of existing software be updated to conform with standards established by the IBSI. Furthermore, it is essential to include in radiomics studies the comprehensive description of feature computation details as defined by the $\operatorname{IBSI}^{65}$ and Vallières et al, ${ }^{146}$ as shown in Table II. Ultimately, we envision the use of dedicated ontologies to improve the interoperability of radiomics analyses via consistent tagging of features, image processing parameters and filters. The Radiomics Ontology (www.bioportal.bioontology.org/ontologies/ $\mathrm{RO}$ ) could provide a standardized means of reporting radiomics data and methods, and would more concisely summarize the implementation details of a given radiomics workflow.

Finally, some guiding principles already exist to help radiomics scientists further implement the responsible research paradigm into their current practice. A concise set of principles for better scientific data management and stewardship, the "FAIR guiding principles,"147 stating that all research objects should be findable, accessible, interoperable, and reusable. Implementation of the FAIR principles within the radiomics field could facilitate its faster clinical translation. First, all methodological details and clinical information must be clearly reported or described to facilitate reproducibility and comparison with other studies and meta-analyses. Second, models must be tested in sufficiently large 
patient datasets distinct from teaching (training and validation) sets to statistically demonstrate their efficacy over conventional models (e.g., existing biomarkers, tumor volume, cancer stage, etc.). To allow for optimal reproducibility potential and further independent testing, all data, final models and programming code related to a given study needs to be made available to the community. Table III provides guidelines that can help to evaluate the quality of radiomics studies. ${ }^{146}$ More guidelines on reproducible prognostic modeling can also be found in the TRIPOD statement. ${ }^{106}$

\section{CONCLUSIONS}

The field of radiomics is constantly growing within the field of medical physics and is an exciting opportunity for the medical physics community to participate in novel research for the safe translation of quantitative imaging. Machine and deep learning-based models have the potential to provide clinicians with DSS to improve diagnosis, treatment selection, and response assessment in oncology. As the field expands, the need to associate radiomic features with other clinical and biological variables will become of increased importance. The field should also continue to strive for standardized data collection, evaluation criteria, and reporting guidelines in order to mature as a field. Data sharing will be crucial to develop the large-scale datasets needed for proper validation of radiomic models and there will be a need for collaborations to validate models across multiple institutions. In order to move radiomic models into the clinical practice it is imperative to demonstrate improvements to the clinical workflow and decision making, through expert observer studies and eventually clinical trials. Future developments in the areas of machine and deep learning with their improved balance of interpretability and prediction will also continue to advance radiomic studies.

\section{AKNOWLEDGEMENTS}

This work was partially supported by institutional startup funds from the University of Michigan as well as NIH/NCI 1R37CA214955-01A1 (to A.R).

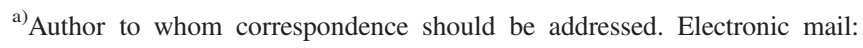
mavanzo@cro.it.

\section{REFERENCES}

1. Avanzo M, Stancanello J, El Naqa I. Beyond imaging: the promise of radiomics. Phys. Med. 2017;38:122-139.

2. Lambin P, Rios-Velazquez E, Leijenaar R, et al. Radiomics: extracting more information from medical images using advanced feature analysis. Eur J Cancer. 2012;48:441-446.

3. Aerts HJ, Velazquez ER, Leijenaar RT, et al. Decoding tumour phenotype by noninvasive imaging using a quantitative radiomics approach. Nat Commun. 2014;5:4006.

4. Panth KM, Leijenaar RT, Carvalho S, et al. Is there a causal relationship between genetic changes and radiomics-based image features? An in vivo preclinical experiment with doxycycline inducible GADD34 tumor cells. Radiother Oncol. 2015;116:462-466.
5. Kumar V, Gu Y, Basu S, et al. Radiomics: the process and the challenges. Magn Reson Imaging. 2012;30:1234-1248.

6. Tang J, Rangayyan RM, Xu J, El Naqa I, Yang Y. Computer-aided detection and diagnosis of breast cancer with mammography: recent advances. IEEE Trans Inf Technol Biomed. 2009;13:236-251.

7. Giger ML, Karssemeijer N, Schnabel JA. Breast image analysis for risk assessment, detection, diagnosis, and treatment of cancer. Аnпи Rev Biomed Eng. 2013;15:327-357.

8. Gillies RJ, Kinahan PE, Hricak H. Radiomics: images are more than pictures, they are data. Radiology. 2016;278:563-577.

9. Wu J, Tha KK, Xing L, Li R. Radiomics and radiogenomics for precision radiotherapy. J Radiat Res. 2018;59:i25-i31.

10. Napel S, Mu W, Jardim-Perassi BV, Aerts HJWL, Gillies RJ. Quantitative imaging of cancer in the postgenomic era: radio(geno)mics, deep learning, and habitats. Cancer. 2018;124:4633-4649.

11. Stoyanova R, Takhar M, Tschudi Y, et al. Prostate cancer radiomics and the promise of radiogenomics. Translat Can Res. 2016;5:432-447.

12. Khalvati F, Wong A, Haider MA. Automated prostate cancer detection via comprehensive multi-parametric magnetic resonance imaging texture feature models. BMC Med Imaging. 2015;15:27.

13. Algohary A, Viswanath S, Shiradkar R, et al. Radiomic features on MRI enable risk categorization of prostate cancer patients on active surveillance: preliminary findings. J Magn Reson Imaging. 2018;48:818-828.

14. Li Q, Bai H, Chen Y, et al. A fully-automatic multiparametric radiomics model: towards reproducible and prognostic imaging signature for prediction of overall survival in glioblastoma multiforme. Sci Rep. 2017; 7:14331.

15. Ibragimov B, Xing L. Segmentation of organs-at-risks in head and neck CT images using convolutional neural networks. Med Phys. 2017;44: $547-557$.

16. Lustberg T, van Soest J, Gooding M, et al. Clinical evaluation of atlas and deep learning based automatic contouring for lung cancer. Radiother Oncol. 2018;126:312-317.

17. Chlebus G, Schenk A, Moltz JH, van Ginneken B, Hahn HK, Meine H. Automatic liver tumor segmentation in CT with fully convolutional neural networks and object-based postprocessing. Sci Rep. 2018;8:15497.

18. Sapate SG, Mahajan A, Talbar SN, Sable N, Desai S, Thakur M. Radiomics based detection and characterization of suspicious lesions on full field digital mammograms. Comput Methods Programs Biomed. 2018; 163:1-20.

19. Antropova N, Huynh BQ, Giger ML. A deep feature fusion methodology for breast cancer diagnosis demonstrated on three imaging modality datasets. Med Phys. 2017;44:5162-5171.

20. Bonekamp D, Kohl S, Wiesenfarth M, et al. Radiomic machine learning for characterization of prostate lesions with MRI: comparison to ADC values. Radiology. 2018;289:128-137.

21. Yuan Y, Qin W, Buyyounouski M, et al. Prostate cancer classification with multiparametric MRI transfer learning model. Med Phys. 2019;46: 756-765.

22. Wu W, Parmar C, Grossmann P, et al. Exploratory study to identify radiomics classifiers for lung cancer histology. Front Oncol. 2016;6:71.

23. Ferreira Junior JR, Koenigkam-Santos M, Cipriano FEG, Fabro AT, Azevedo-Marques PM. Radiomics-based features for pattern recognition of lung cancer histopathology and metastases. Comput Methods Programs Biomed. 2018;159:23-30.

24. Ganeshan B, Abaleke S, Young RC, Chatwin CR, Miles KA. Texture analysis of non-small cell lung cancer on unenhanced computed tomography: initial evidence for a relationship with tumour glucose metabolism and stage. Cancer Imaging. 2010;10:137-143.

25. Song SH, Park H, Lee G, et al. Imaging phenotyping using radiomics to predict micropapillary pattern within lung adenocarcinoma. J Thorac Oncol. 2017;12:624-632.

26. Crispin-Ortuzar M, Apte A, Grkovski M, et al. Predicting hypoxia status using a combination of contrast-enhanced computed tomography and [(18)F]-Fluorodeoxyglucose positron emission tomography radiomics features. Radiother Oncol. 2018;127:36-42.

27. Parra AN, Lu H, Li Q, et al. Predicting clinically significant prostate cancer using DCE-MRI habitat descriptors. Oncotarget. 2018;9:3712537136.

28. Lee J, Narang S, Martinez JJ, Rao G, Rao A. Associating spatial diversity features of radiologically defined tumor habitats with epidermal 
growth factor receptor driver status and 12-month survival in glioblastoma: methods and preliminary investigation. J Med Imaging (Bellingham). 2015;2:041006.

29. Zhu Y, Li H, Guo W, et al. Deciphering genomic underpinnings of quantitative MRI-based radiomic phenotypes of invasive breast carcinoma. Sci Rep. 2015;5:17787.

30. Liu J, Kim Y, Balagurunathan Q, et al. Radiomic features are associated with EGFR mutation status in lung adenocarcinomas. Clin Lung Cancer. 2016;17:441-448.

31. Yoon HJ, Sohn I, Cho JH, et al. Decoding tumor phenotypes for ALK, ROS1, and RET fusions in lung adenocarcinoma using a radiomics approach. Medicine (Baltimore). 2015;94:e1753.

32. Wang J, Kato F, Oyama-Manabe N, et al. Identifying triple-negative breast cancer using background parenchymal enhancement heterogeneity on dynamic contrast-enhanced MRI: A Pilot Radiomics Study. PLoS ONE. 2015;10:e0143308.

33. Lee SE, Han K, Kwak JY, Lee E, Kim EK. Radiomics of US texture features in differential diagnosis between triple-negative breast cancer and fibroadenoma. Sci Rep. 2018;8:13546.

34. Diehn M, Nardini C, Wang DS, et al. Identification of noninvasive imaging surrogates for brain tumor gene-expression modules. Proc Natl Acad Sci USA. 2007;105:5213-5218.

35. Gnep K, Fargeas A, Gutierrez-Carvajal RE, et al. Haralick textural features on T2 -weighted MRI are associated with biochemical recurrence following radiotherapy for peripheral zone prostate cancer. J Magn Reson Imaging. 2016;45:103-117.

36. Bogowicz M, Riesterer O, Ikenberg K, et al. Computed tomography radiomics predicts HPV status and local tumor control after definitive radiochemotherapy in head and neck squamous cell carcinoma. Int $J$ Radiat Oncol Biol Phys. 2017;99:921-928.

37. Leijenaar RT, Bogowicz M, Jochems A, et al. Development and validation of a radiomic signature to predict HPV (p16) status from standard CT imaging: a multicenter study. Br J Radiol. 2018;91:20170498.

38. Nie K, Shi L, Chen Q, et al. Rectal cancer: assessment of neoadjuvant chemo-radiation outcome based on radiomics of multi-parametric MRI. Clin Cancer Res. 2016;22:5256-5263.

39. Oikonomou A, Khalvati F, Tyrrell PN, et al. Radiomics analysis at PET/ CT contributes to prognosis of recurrence and survival in lung cancer treated with stereotactic body radiotherapy. Sci Rep. 2018;8:4003.

40. Liu J, Mao Y, Li Z, et al. Use of texture analysis based on contrast-enhanced MRI to predict treatment response to chemoradiotherapy in nasopharyngeal carcinoma. J Magn Reson Imaging. 2016;44:445-455.

41. Ibragimov B, Toesca D, Yuan Y, Koong A, Daniel C, Xing L. Neural networks for deep radiotherapy dose analysis and prediction of liver SBRT outcomes. IEEE J Biomed Health Inform. 2019:1-1. https://doi. org/10.1109/JBHI.2019.2904078

42. Huynh E, Coroller TP, Narayan V, et al. CT-based radiomic analysis of stereotactic body radiation therapy patients with lung cancer. Radiother Oncol. 2016;120:258-266.

43. Vallieres M, Freeman CR, Skamene SR, El Naqa I. A radiomics model from joint FDG-PET and MRI texture features for the prediction of lung metastases in soft-tissue sarcomas of the extremities. Phys Med Biol. 2015;60:5471-5496.

44. Coroller TP, Grossmann P, Hou Y, et al. CT-based radiomic signature predicts distant metastasis in lung adenocarcinoma. Radiother Oncol. 2015; 114:345-350.

45. Hao H, Zhou Z, Li S, et al. Shell feature: a new radiomics descriptor for predicting distant failure after radiotherapy in non-small cell lung cancer and cervix cancer. Phys Med Biol. 2018;63:095007.

46. Haralick RM, Shanmugam K, Dinstein I. Textural features for image classification. IEEE Trans Syst Man Cybern. 1973;3:610-621.

47. Ohri N, Duan F, Snyder BS, et al. Pretreatment 18F-FDG PET textural features in locally advanced non-small cell lung cancer: secondary analysis of ACRIN 6668/RTOG 0235. J Nucl Med. 2016;57:842-848.

48. Hosny A, Parmar C, Coroller TP, et al. Deep learning for lung cancer prognostication: a retrospective multi-cohort radiomics study. PLoS Medicine. 2018; 15:e1002711.

49. Aerts HJ. The potential of radiomic-based phenotyping in precision medicine: a review. JAMA Oncol. 2016;2:1636-1642.

50. Kickingereder P, Gotz M, Muschelli J, et al. Large-scale radiomic profiling of recurrent glioblastoma identifies an imaging predictor for stratifying anti-angiogenic treatment response. Clin Cancer Res. 2016;22:5765-5771.

51. Sun R, Limkin EJ, Vakalopoulou M, et al. A radiomics approach to assess tumour-infiltrating CD8 cells and response to anti-PD-1 or antiPD-L1 immunotherapy: an imaging biomarker, retrospective multicohort study. Lancet Oncol. 2018;19:1180-1191.

52. Fave X, Zhang L, Yang J, et al. Delta-radiomics features for the prediction of patient outcomes in non-small cell lung cancer. Sci Rep. 2017;7:588.

53. Fave X, Mackin D, Yang J, et al. Can radiomics features be reproducibly measured from CBCT images for patients with non-small cell lung cancer? Med Phys. 2015;42:6784-6797.

54. van Timmeren JE, Leijenaar RT, van Elmpt W, Lambin P. Interchangeability of a radiomic signature between conventional and weekly cone beam computed tomography allowing response prediction in non-small cell lung cancer. Int J Radiat Oncol Biol Phys. 2016;96:S193.

55. van Timmeren JE, Leijenaar RTH, van Elmpt W, Reymen B, Lambin P. Feature selection methodology for longitudinal cone-beam CT radiomics. Acta Oncol. 2017;56:1537-1543.

56. Moran A, Daly ME, Yip SSF, Yamamoto T. Radiomics-based assessment of radiation-induced lung injury after stereotactic body radiotherapy. Clin. Lung Cancer. 2017;18:e425-e431.

57. Cunliffe A, Armato SG 3rd, Castillo R, Pham N, Guerrero T, Al-Hallaq HA. Lung texture in serial thoracic computed tomography scans: correlation of radiomics-based features with radiation therapy dose and radiation pneumonitis development. Int $J$ Radiat Oncol Biol Phys. 2015;91:1048-1056.

58. Krafft SP, Rao A, Stingo F, et al. The utility of quantitative CT radiomics features for improved prediction of radiation pneumonitis. Med Phys. 2018;45:5317-5324.

59. Mattonen SA, Palma DA, Johnson C, et al. Detection of local cancer recurrence after stereotactic ablative radiation therapy for lung cancer: physician performance versus radiomic assessment. Int J Radiat Oncol Biol Phys. 2016;94:1121-1128.

60. Zhang Z, Yang J, Ho A, et al. A predictive model for distinguishing radiation necrosis from tumour progression after gamma knife radiosurgery based on radiomic features from MR images. Eur Radiol. 2018;28:2255-2263.

61. Viswanath S, Toth R, Rusu M, et al. Identifying quantitative in vivo multi-parametric mri features for treatment related changes after laser interstitial thermal therapy of prostate cancer. Neurocomputing. 2014;144:13-23.

62. Yu JY, Zhang HP, Tang ZY, et al. Value of texture analysis based on enhanced MRI for predicting an early therapeutic response to transcatheter arterial chemoembolisation combined with high-intensity focused ultrasound treatment in hepatocellular carcinoma. Clin Radiol. 2018;73:758.e9-758.e18.

63. Bagher-Ebadian H, Siddiqui F, Liu C, Movsas B, Chetty IJ. On the impact of smoothing and noise on robustness of CT and CBCT radiomics features for patients with head and neck cancers. Med Phys. 2017;44:1755-1770.

64. Shafiq-Ul-Hassan M, Zhang GG, Latifi K, et al. Intrinsic dependencies of CT radiomic features on voxel size and number of gray levels. Med Phys. 2017;44:1050-1062.

65. Alex Z, Stefan L, Martin V, Steffen L. The Image Biomarker Standardisation Initiative for, "Image biomarker standardisation initiative," arXiv e-prints, ar:1612.07003; 2016.

66. van Velden FH, Kramer GM, Frings V, et al. Repeatability of radiomic features in non-small-cell lung cancer [18F]FDG-PET/CT studies: impact of reconstruction and delineation. Mol Imaging Biol. 2016;18:788-795.

67. Leijenaar RT, Nalbantov G, Carvalho S, et al. The effect of SUV discretization in quantitative FDG-PET Radiomics: the need for standardized methodology in tumor texture analysis. Sci Rep. 2015;5:11075.

68. Julesz B. Experiments in the visual perception of texture. Sci Am. 1975;232:34-43.

69. El Naqa I, Grigsby P, Apte A, et al. Exploring feature-based approaches in PET images for predicting cancer treatment outcomes. Pattern Recognit. 2009;42:1162-1171.

70. Coroller TP, Agrawal V, Narayan V, et al. Radiomic phenotype features predict pathological response in non-small cell lung cancer. Radiother Oncol. 2016;119:480-486. 
71. Tixier F, Hatt M, Valla C, et al. Visual versus quantitative assessment of intratumor 18F-FDG PET uptake heterogeneity: prognostic value in non-small cell lung cancer. J Nucl Med. 2014;55:1235-1241.

72. Parmar C, Grossmann P, Bussink J, Lambin P, Aerts HJ. Machine learning methods for quantitative radiomic biomarkers. Sci Rep. 2015;5:13087.

73. Tibshirani R. Regression shrinkage and selection via the lasso. J R Stat Soc. 1996;58:267-288.

74. Wold S, Esbensen K, Geladi P. Principal component analysis. Chemometr Intell Lab Syst. 1987;2:37-52.

75. van der Maaten L, Hinton GE. Visualizing data using t-SNE. $J$ Mach Learn Res. 2008;9:2579-2605.

76. Parmar C, Leijenaar RTH, Grossmann P, et al. Radiomic feature clusters and prognostic signatures specific for lung and head \& neck cancer. Sci Rep. 2015;5:11044.

77. Wu J, Cui Y, Sun X, et al. Unsupervised clustering of quantitative image phenotypes reveals breast cancer subtypes with distinct prognoses and molecular pathways. Clin Cancer Res. 2017;23:3334-3342.

78. Ypsilantis PP, Siddique M, Sohn HM, et al. Predicting response to neoadjuvant chemotherapy with PET imaging using convolutional neural networks. PLOS ONE. 2015;10:e137036.

79. Chen S, Zhou S, Yin FF, Marks LB, Das SK. Investigation of the support vector machine algorithm to predict lung radiation-induced pneumonitis. Med. Phys. 2007;34:3808-3814.

80. El Naqa I, Li R, Murphy MJ, eds. Machine Learning in Radiation Oncology: Theory and Application. Cham, Switzerland: Springer International Publishing; 2015.

81. Yang D, Rao G, Martinez J, Veeraraghavan A, Rao A. Evaluation of tumor-derived MRI-texture features for discrimination of molecular subtypes and prediction of 12-month survival status in glioblastoma. Med Phys. 2015;42:6725-6735.

82. Vallieres M, Kay-Rivest E, Perrin L, et al. Radiomics strategies for risk assessment of tumour failure in head-and-neck cancer. Sci Rep. 2017;7:10117.

83. Rosenberg C, Hebert M, Schneiderman H. Semi-Supervised Self-Training of Object Detection Models. In: WACV-MOTION '05 Proceedings of the Seventh IEEE Workshops on Application of Computer Vision (WACV/MOTION'05), Volume 1, 29-36; 2005. https://doi.org/10. 1109/ACVMOT.2005.107

84. Joachims T. Transductive Inference for Text Classification Using Support Vector Machines. In: ICML ' 99 Proceedings of the Sixteenth International Conference on Machine Learning, 200-209; 1999. https://doi. org/10.1109/ACVMOT.2005.107

85. Blum A, Lafferty J, Rwebangira MR, Reddy R. Semi-supervised Learning Using Randomized Mincuts. In: ICML '04 Proceedings of the twenty-first international conference on Machine learning, 13; 2004. https://doi.org/10.1145/1015330.1015429

86. Cox DR. Regression models and life-tables. J R Stat Soc. 1972;34:187220.

87. Ishwaran H, Kogalur UB, Blackstone EH, Lauer MS. Random survival forests. Ann Appl Stat. 2008;2:841-860.

88. Van Belle V, Pelckmans K, Van Huffel S, Suykens JAK. Support vector methods for survival analysis: a comparison between ranking and regression approaches. Artif Intell Med. 2011;53:107-118.

89. LeCun Y, Bengio Y, Hinton G. Deep learning. Nature. 2015;521:436444.

90. Suzuki K. Pixel-based machine learning in medical imaging. Int $J$ Biomed Imaging. 2012;2012:792079.

91. Simonyan K, Zisserman A. Very Deep Convolutional Networks for Large-Scale Image Recognition. arXiv:1409.1556; 2014.

92. He K, Zhang X, Ren S, Sun J. Deep Residual Learning for Image Recognition. Presented at 2016 IEEE Conference on Computer Vision and Pattern Recognition (CVPR) 2016. 770-778; 2016.

93. Hubel DH, Wiesel TN. Receptive fields and functional architecture of monkey striate cortex. J Physiol (Lond.). 1968;195:215-243.

94. Hochreiter S, Schmidhuber J. Long short-term memory. Neural Comput. 1997;9:1735-1780.

95. Cho K, van Merrienboer B, Gulcehre C, et al.Learning Phrase Representations using RNN Encoder-Decoder for Statistical Machine Translation. In: Proceedings of the 2014 Conference on Empirical Methods in Natural Language Processing (EMNLP), 1724-1734; 2014. https://d oi.org/10.3115/v1/D14-1179
96. Kingma DP, Welling M. Auto-Encoding Variational Bayes. arXiv: $1312.6114 ; 2013$.

97. Li F, Qiao H, Zhang B. Discriminatively boosted image clustering with fully convolutional auto-encoders. Pattern Recogn. 2018;83:161-173.

98. Hinton GE, Salakhutdinov RR. Reducing the dimensionality of data with neural networks. Science. 2006;313:504-507.

99. Kingma DP, Rezende DJ, Mohamed S, Welling M. Semi-Supervised Learning with Deep Generative Models. eprint arXiv:1406.5298; 2014.

100. Hinton GE, Srivastava N, Krizhevsky A, Sutskever I, Salakhutdinov RR. Improving neural networks by preventing co-adaptation of feature detectors. arXiv e-prints, ar:1207.0580; 2012.

101. Sergey I, Christian S. Batch Normalization: Accelerating Deep Network Training by Reducing Internal Covariate Shift. arXiv e-prints, ar: $1502.03167 ; 2015$.

102. Long J, Shelhamer E, Darrell T. Fully convolutional networks for semantic segmentation. IEEE Trans Pattern Anal Mach Intell. 2017;39: 640-651.

103. Ronneberger O, Fischer P, Brox T. U-Net: Convolutional Networks for Biomedical Image Segmentation. Presented at Medical Image Computing and Computer-Assisted Intervention (MICCAI); 2015. https://doi. org/10.1007/978-3-319-24574-4_28

104. Esteva A, Kuprel B, Novoa RA, et al. Dermatologist-level classification of skin cancer with deep neural networks. Nature. 2017;542: $115-118$.

105. Chang H, Han J, Zhong C, Snijders AM, Mao JH. Unsupervised transfer learning via multi-scale convolutional sparse coding for biomedical applications. IEEE Trans Pattern Anal Mach Intell. 2018;40:1182-1194.

106. Collins GS, Reitsma JB, Altman DG, Moons KG. Transparent reporting of a multivariable prediction model for individual prognosis or diagnosis (TRIPOD): the TRIPOD statement. BMC Med. 2015;13:W1.

107. Hastie T, Tibshirani R, Friedman JH. The Elements of Statistical Learning: Data Mining, Inference, and Prediction. New York, NY: Springer; 2009.

108. Ren J, Zhou J, Ding W, Zhong B, Zhou J. Clinicopathological characteristics and imaging features of pulmonary adenocarcinoma with micropapillary pattern. Zhonghua Zhong Liu Za Zhi. 2014;36:282-286.

109. Harrell FE Jr, Lee KL, Mark DB. Multivariable prognostic models: issues in developing models, evaluating assumptions and adequacy, and measuring and reducing errors. Stat Med. 1996;15:361-387.

110. Pencina MJ, D'Agostino RB. Overall C as a measure of discrimination in survival analysis: model specific population value and confidence interval estimation. Statist Med. 2004;23:2109-2123.

111. Royston P, Altman DG. External validation of a Cox prognostic model: principles and methods. BMC Med Res Methodol. 2013;13:33.

112. Boellaard R. Standards for PET image acquisition and quantitative data analysis. J Nucl Med. 2009;50:11S-20S.

113. Mackin D, Fave X, Zhang L, et al. Measuring computed tomography scanner variability of radiomics features. Invest Radiol. 2015;50:757765 .

114. Szczypiński PM, Strzelecki M, Materka A, Klepaczko A. MaZda-A software package for image texture analysis. Comput Methods Progr Biomed. 2009;94:66-76.

115. Nioche C, Orlhac F, Boughdad S, et al. LIFEx: a freeware for radiomic feature calculation in multimodality imaging to accelerate advances in the characterization of tumor heterogeneity. Cancer Res. 2018;78:47864789 .

116. Rubin DL, Willrett D, O'Connor MJ, Hage C, Kurtz C, Moreira DA. Automated tracking of quantitative assessments of tumor burden in clinical trials. Transl Oncol. 2014;7:23-35.

117. van Griethuysen JJM, Fedorov A, Parmar C, et al. Computational radiomics system to decode the radiographic phenotype. Cancer Res. 2017;77:e104-e107.

118. Dicente Cid Y, Castelli J, Schaer R, Scher N, Pomoni A, Prior JO, Depeursinge A. Chapter 12 - QuantImage: An Online Tool for HighThroughput 3D Radiomics Feature Extraction in PET-CT. In: Biomedical Texture Analysis, 349-377; 2017. https://doi.org/10.1016/B978-012-812133-7.00012-0

119. Echegaray S, Bakr S, Rubin DL, Napel S. Quantitative image feature engine (QIFE): an open-source, modular engine for 3D quantitative 
feature extraction from volumetric medical images. J Digit Imaging. 2018;31:403-414.

120. Zhang L, Fried DV, Fave XJ, Hunter LA, Yang J, Court LE. IBEX: an open infrastructure software platform to facilitate collaborative work in radiomics. Med Phys. 2015;42:1341-1353.

121. Fedorov A, Beichel R, Kalpathy-Cramer J, et al. 3D slicer as an image computing platform for the quantitative imaging network. Magn Reson Imaging. 2012;30:1323-1341.

122. Yosinski J, Clune J, Nguyen A, Fuchs T, Lipson H.Understanding Neural Networks Through Deep Visualization. eprint arXiv:1506.06579; 2015.

123. Sankar V, Kumar D, Clausi DA, Taylor GW, Wong A. SISC: End-toend Interpretable Discovery Radiomics-Driven Lung Cancer Prediction via Stacked Interpretable Sequencing Cells. eprint arXiv:1901.04641; 2019.

124. Luo Y, McShan D, Ray D, et al. Development of a fully cross-validated bayesian network approach for local control prediction in lung cancer. IEEE Trans Radiat Plasma Med Sci. 2019;3:232-241.

125. Hatt M, Tixier F, Pierce L, Kinahan PE, Le Rest CC, Visvikis D. Characterization of PET/CT images using texture analysis: the past, the present.. any future? Eur J Nucl Med Mol Imaging. 2017;44: $151-165$.

126. Fave X, Cook M, Frederick A, et al. Preliminary investigation into sources of uncertainty in quantitative imaging features. Comput Med Imaging Graph. 2015;44:54-61.

127. Pfaehler E, Beukinga RJ, de Jong JR, et al. Repeatability of (18) FFDG PET radiomic features: a phantom study to explore sensitivity to image reconstruction settings, noise, and delineation method. Med Phys. 2019;46:665-678.

128. Leijenaar RT, Carvalho S, Velazquez ER, et al. Stability of FDG-PET radiomics features: an integrated analysis of test-retest and inter-observer variability. Acta Oncol. 2013;52:1391-1397.

129. Kim H, Park CM, Lee M, et al. Impact of reconstruction algorithms on CT radiomic features of pulmonary tumors: analysis of intra- and interreader variability and inter-reconstruction algorithm variability. PLoS ONE. 2016;11:e0164924.

130. Zhao B, James LP, Moskowitz CS, et al. Evaluating variability in tumor measurements from same-day repeat CT scans of patients with nonsmall cell lung cancer. Radiology. 2009;252:263-272.

131. Desseroit MC, Tixier F, Weber WA, et al. Reliability of PET/CT shape and heterogeneity features in functional and morphological components of non-small cell lung cancer tumors: a repeatability analysis in a prospective multi-center cohort. J Nucl Med. 2016;58: 406-411.

132. Galavis PE, Hollensen C, Jallow N, Paliwal B, Jeraj R. Variability of textural features in FDG PET images due to different acquisition modes and reconstruction parameters. Acta Oncol. 2010;49:1012-1016.
133. Lu L, Lv W, Jiang J, et al. Robustness of radiomic features in [11C] Choline and [18F]FDG PET/CT imaging of nasopharyngeal carcinoma: impact of segmentation and discretization. Mol Imaging Biol. 2016;18:935-945.

134. Bailly C, Bodet-Milin C, Couespel S, et al. Revisiting the robustness of PET-based textural features in the context of multi-centric trials. PLoS ONE. 2016;11:e0159984.

135. Yang F, Dogan N, Stoyanova R, Ford JC. Evaluation of radiomic texture feature error due to MRI acquisition and reconstruction: a simulation study utilizing ground truth. Phys Med. 2018;50:26-36.

136. Grootjans W, Tixier F, van der Vos CS, et al. The impact of optimal respiratory gating and image noise on evaluation of intra-tumor heterogeneity in 18F-FDG positron emission tomography imaging of lung cancer. J Nucl Med. 2016;57:1692-1698.

137. Parmar C, Rios Velazquez E, Leijenaar R, et al. Robust radiomics feature quantification using semiautomatic volumetric segmentation. PLoS ONE. 2014;9:e102107.

138. Echegaray S, Gevaert O, Shah R, et al. Core samples for radiomics features that are insensitive to tumor segmentation: method and pilot study using CT images of hepatocellular carcinoma. J Med Imaging (Bellingham). 2015;2:041011.

139. Lambin P, Leijenaar RTH, Deist TM, et al. Radiomics: the bridge between medical imaging and personalized medicine. Nat Rev Clin Oncol. 2017;14:749-762.

140. Chalkidou A, O'Doherty MJ, Marsden PK. False discovery rates in PET and CT studies with texture features: a systematic review. PLoS ONE. 2015;10:e0124165.

141. AlBadawy EA, Saha A, Mazurowski MA. Deep learning for segmentation of brain tumors: impact of cross-institutional training and testing. Med Phys. 2018;45:1150-1158.

142. Johnson WE, Li C, Rabinovic A. Adjusting batch effects in microarray expression data using empirical Bayes methods. Biostatistics. 2007;8:118-127.

143. Chang K, Balachandar N, Lam C, et al. Distributed deep learning networks among institutions for medical imaging. J Am Med Inform Assoc. 2018;25:945-954.

144. Zur RM, Jiang Y, Pesce LL, Drukker K. Noise injection for training artificial neural networks: a comparison with weight decay and early stopping. Med Phys. 2009;36:4810-4818.

145. Li S, Chen Y, Peng Y, Bai L. Learning More Robust Features with Adversarial Training. arXiv:1804.07757; 2018.

146. Vallieres M, Zwanenburg A, Badic B, Cheze Le Rest C, Visvikis D, Hatt M. Responsible radiomics research for faster clinical translation. $J$ Nucl Med. 2018;59:189-193.

147. Wilkinson MD, Dumontier M, Aalbersberg IJ, et al. The FAIR guiding principles for scientific data management and stewardship. Sci Data. 2016:3:160018. 